\title{
On Von Neumann's Inequality for Matrices of Complex Polynomials
}

\author{
Joachim Moussounda Mouanda \\ Mathematics Department, Blessington Christian University, Nkayi, Republic of Congo \\ Email: mmoussounda@yahoo.fr
}

How to cite this paper: Mouanda, J.M. (2021) On Von Neumann's Inequality for Matrices of Complex Polynomials. American Journal of Computational Mathematics, 11, 289-303.

https://doi.org/10.4236/ajcm.2021.114019

Received: September 15, 2021

Accepted: December 7, 2021

Published: December 10, 2021

Copyright (c) 2021 by author(s) and Scientific Research Publishing Inc. This work is licensed under the Creative Commons Attribution International License (CC BY 4.0).

http://creativecommons.org/licenses/by/4.0/

\begin{abstract}
We prove that every matrix $F \in M_{k}\left(\mathcal{P}_{n}\right)$ is associated with the smallest positive integer $d(F) \neq 1$ such that $d(F)\|F\|_{\infty}$ is always bigger than the sum of the operator norms of the Fourier coefficients of $F$. We establish some inequalities for matrices of complex polynomials. In application, we show that von Neumann's inequality holds up to the constant $2^{n}$ for matrices of the algebra $M_{k}\left(\mathcal{P}_{n}\right)$.
\end{abstract}

\section{Keywords}

Fourier Coefficients, Operator Theory, Polynomials

\section{Introduction}

In 1951, von Neumann [1] showed that for any contraction linear operator $\mathrm{T}$ on a Hilbert space the inequality

$$
\|p(T)\| \leq\|p\|_{\infty},
$$

holds for all complex polynomials $p(z)$ over the unit disk, where $\|p\|_{\infty}$ denotes the supremum norm of $p$ over the unit disk. This result was generalised by many people. In particular, Brehmer [2] proved in 1961 that von Neumann's inequality also holds for families $\left\{S_{1}, S_{2}, \cdots, S_{n}\right\}$ of commuting operators on a complex Hilbert space with

$$
\sum_{i=1}^{n}\left\|S_{i}\right\|^{2} \leq 1
$$

In 1963, Ando [3] established the natural generalisation of von Neumann's inequality for polynomials in two commuting contractions. In 1974, Varopoulos [4] proved that the analogue of von Neumann's inequality fails for 3 or more 
commuting contractions. There are several such counterexamples in the literature [5]. In 1978, Lubin [6] proved that if $T_{1}, \cdots, T_{n}$ are commuting contractions on a Hilbert space, then

$$
\left\|p\left(T_{1}, \cdots, T_{n}\right)\right\| \leq \sup \left\{\left|p\left(z_{1}, \cdots, z_{n}\right)\right|:\left|z_{i}\right|<\sqrt{n}\right\},
$$

for any polynomial $p\left(z_{1}, \cdots, z_{n}\right)$ over $\mathbb{D}^{n}$. von Neumann's inequality holds for commutative families of isometries and doubly commuting sets of contractions [7]. Recent work of Kosiński on the three point Pick interpolation problem on polydisc shows that von Neumann's inequality holds for $3 \times 3$ commuting contractive matrices [8]. In 2020, Mouanda proved that von Neumann's inequality holds for $n$-tuples of upper (or lower) complex triangular Toeplitz (or circulant) contractions [9]. This result was first extended to matrices of complex polynomials in 2021 by Mouanda [10].

This result, which has many engineering applications, is a fundamental tool in operator theory [5] [11].

In this paper, we are mainly concerned with the following long-standing question: Does von Neumann's inequality hold up to some constant for $n$-tuples of commuting contractions? First of all, we show that every $F \in M_{k}\left(\mathcal{P}_{n}\right)$ is associated with the smallest positive integer $d(F) \neq 1$ such that $d(F)\|F\|_{\infty}$ is always bigger than the sum of the operator norms of the Fourier coefficients of $F$. We establish some inequalities for matrices of complex polynomials.

Theorem 1.1. Let $\mathcal{P}_{n}$ be the algebra of complex polynomials over $\mathbb{D}^{n}$, let $F \in M_{k}\left(\mathcal{P}_{n}\right)$ be a matrix of complex polynomials and let $\left\{T_{1}, T_{2}, \cdots, T_{n}\right\}$ be a set of commuting contractions on the Hilbert space $\mathcal{H}$. Then there exists a sequence $\left(\alpha_{m}(F)\right)_{m \in \mathbb{N}, m \neq 0}$ of positive numbers such that

$$
1 \leq \frac{m\left\|F\left(T_{1}, \cdots, T_{n}\right)\right\|+\alpha_{m}(F)}{m\|F\|_{\infty}+\alpha_{m}(F)} \leq 2^{n}, m \in \mathbb{N}, m \neq 0,
$$

with

$$
\alpha_{m}(F)=\left(2^{n-1}-1\right)\left\|\sum_{\left(k_{1}, \cdots, k_{n}\right) \in S_{F}}\left|m \hat{F}\left(k_{1}, \cdots, k_{n}\right)+\alpha_{m}\left(k_{1}, \cdots, k_{n}\right) I_{k}\right|^{2}\right\|_{M_{k}}^{n-1}+\varphi_{m}(F)
$$

where $\alpha_{m}\left(k_{1}, \cdots, k_{n}\right) \neq 0$ is a positive integer such that

$$
\begin{gathered}
m\left\|\hat{F}\left(k_{1}, \cdots, k_{n}\right)\right\|_{M_{k}}<\left\|m \hat{F}\left(k_{1}, \cdots, k_{n}\right)+\left.\alpha_{m}\left(k_{1}, \cdots, k_{n}\right) I_{k}\right|^{2}\right\|_{M_{k}}, \\
\sum_{\left(k_{1}, \cdots, k_{n}\right) \in S_{f}} m\left\|\hat{F}\left(k_{1}, \cdots, k_{n}\right)\right\|_{M_{k}}<\varphi_{m}(f)
\end{gathered}
$$

and

$$
\varphi_{m}(f)<\left\|\sum_{\left(k_{1}, \cdots, k_{n}\right) \in S_{F}}\left|m \hat{F}\left(k_{1}, \cdots, k_{n}\right)+\alpha_{m}\left(k_{1}, \cdots, k_{n}\right) I_{k}\right|^{2}\right\|_{M_{k}}^{n-1} .
$$

In application, we show that the von Neumann inequality holds up to the constant $2^{n}$ for matrices of the algebra $M_{k}\left(\mathcal{P}_{n}\right)$. 


\section{Proof of the Main Result}

In this section, we give the proof of Theorem 1.1. Given

$$
f\left(z_{1}, \cdots, z_{n}\right)=\sum_{\left(k_{1}, \cdots, k_{n}\right) \in S_{f}} \hat{f}\left(k_{1}, \cdots, k_{n}\right) z_{1}^{k_{1}} \cdots z_{n}^{k_{n}},
$$

a complex polynomial over $\mathbb{D}^{n}$. Then

$$
\|f\|_{\infty}=\sup \left\{\left|f\left(z_{1}, \cdots, z_{n}\right)\right|:\left|z_{1}\right|=\cdots=\left|z_{n}\right|=1\right\} .
$$

Suppose that

$$
f\left(z_{1}, z_{2}\right)=5 z_{1}+28 z_{1} z_{2}+15, g\left(z_{1}, z_{2}\right)=3 z_{1} z_{2}+1, h\left(z_{1}, z_{2}\right)=4 z_{1}+6 z_{1} z_{2}+5
$$

are complex polynomials over $\mathbb{D}^{2}$. Then the $2 \times 2$-matrix

$$
F\left(z_{1}, z_{2}\right)=\left(\begin{array}{ll}
f\left(z_{1}, z_{2}\right) & g\left(z_{1}, z_{2}\right) \\
h\left(z_{1}, z_{2}\right) & f\left(z_{1}, z_{2}\right)
\end{array}\right)
$$

can be written as

$$
F\left(z_{1}, z_{2}\right)=\left(\begin{array}{cc}
15 & 1 \\
5 & 15
\end{array}\right)+\left(\begin{array}{cc}
5 & 0 \\
4 & 5
\end{array}\right) z_{1}+\left(\begin{array}{cc}
28 & 3 \\
6 & 28
\end{array}\right) z_{1} z_{2} .
$$

$F\left(z_{1}, z_{2}\right)$ is a polynomial over $\mathbb{D}^{2}$ with matrix coefficients. Let $\mathcal{P}_{n}$ denote the algebra of complex polynomials over $\mathbb{D}^{n}$. Given

$$
f_{i, j}\left(z_{1}, \cdots, z_{n}\right)=\sum_{\left(k_{1}, \cdots, k_{n}\right) \in S_{f_{i, j}}} \hat{f}_{i, j}\left(k_{1}, \cdots, k_{n}\right) z_{1}^{k_{1}} \cdots z_{n}^{k_{n}}, i, j=1, \cdots, k ;
$$

complex polynomials over $\mathbb{D}^{n}$. Then

$$
\left\|\left(f_{i, j}\right)_{i, j=1}^{k}\right\|_{\infty}=\sup \left\{\left\|\left(f_{i, j}\left(z_{1}, \cdots, z_{n}\right)\right)_{i, j=1}^{k}\right\|_{M_{k}}:\left|z_{i}\right| \leq 1\right\} .
$$

Denote by

$$
S_{F}=\bigcup_{i=1}^{k} \bigcup_{j=1}^{k} S_{f_{i, j}}
$$

Then the $k \times k$-matrix $F\left(z_{1}, \cdots, z_{n}\right)=\left(f_{i, j}\left(z_{1}, \cdots, z_{n}\right)\right)_{i, j=1}^{k}$ can be written as

$$
F\left(z_{1}, \cdots, z_{n}\right)=\sum_{\left(k_{1}, \cdots, k_{n}\right) \in S_{F}}\left(\hat{f}_{i, j}\left(k_{1}, \cdots, k_{n}\right)\right)_{i, j=1}^{k} z_{1}^{k_{1}} \cdots z_{n}^{k_{n}} .
$$

In other words,

$$
F\left(z_{1}, \cdots, z_{n}\right)=\sum_{\left(k_{1}, \cdots, k_{n}\right) \in S_{F}} \hat{F}\left(k_{1}, \cdots, k_{n}\right) z_{1}^{k_{1}} \cdots z_{n}^{k_{n}} .
$$

The $k \times k$-matrix $F\left(z_{1}, \cdots, z_{n}\right)$ can be regarded as a polynomial over $\mathbb{D}^{n}$ with matrix coefficients. Each element of the algebra $M_{k}\left(\mathcal{P}_{n}\right)$ has this representation. In our case, the set

$$
\left\{\hat{F}\left(k_{1}, \cdots, k_{n}\right):\left(k_{1}, \cdots, k_{n}\right) \in S_{F}\right\}
$$

is the set of Fourier coefficients of $F\left(z_{1}, \cdots, z_{n}\right)$. Let us set

$$
\|F\|_{u, k}=\sup \left\|\left(f_{i, j}\left(T_{1}, \cdots, T_{n}\right)\right)_{i, j=1}^{k}\right\|
$$


where the supremum is taken over the family of all $n$-tuples of commuting contractions on all Hilbert spaces. It is easy to see that $\|F\|_{u, k}$ is finite, since it is bounded by the sum of the operator norms of the Fourier coefficients of $F$, and that this quantity defines a norm on the algebra $M_{k}\left(\mathcal{P}_{n}\right)$ of matrices of polynomials over $\mathbb{D}^{n}$. For each polynomial $P$ in $M_{k}\left(\mathcal{P}_{n}\right)$, there is always an $n$-tuple of contractions where this supremum is achieved. Therefore, $\left(M_{k}\left(\mathcal{P}_{n}\right),\|\|_{u, k}\right)$ is a normed algebra.

Definition 2.1. Let $G$ be a group. An order $>$, on $G$, is called archimedean if it has the following property: to every pair of elements $x, y$ of $G$ such that $x>0$ and $y>0$, there corresponds a positive integer $n$ such that $n x>y$.

The order axiom for the real line states that every real number is less than some natural number. This is equivalent to the assertion that for any two positive real numbers $a$ and $b$ there is a positive integer $n$ such that $a \leq n b$.

The Archimedean property of the total order on $\mathbb{R}$ allows us to show that every element $F\left(z_{1}, \cdots, z_{n}\right)$ of $M_{k}\left(\mathcal{P}_{n}\right)$ is associated to the smallest positive integer $d(F) \neq 1$ such that $d(F)\|F\|_{\infty}$ is always bigger than the sum of the operator norms of the Fourier coefficients of $F\left(z_{1}, \cdots, z_{n}\right)$.

Theorem 2.2. Let

$$
F\left(z_{1}, \cdots, z_{n}\right)=\sum_{\left(k_{1}, \cdots, k_{n}\right) \in S_{F}} \hat{F}\left(k_{1}, \cdots, k_{n}\right) z_{1}^{k_{1}} \cdots z_{n}^{k_{n}}
$$

be an element of $M_{k}\left(\mathcal{P}_{n}\right)$. Then there exists a smallest positive interger $d(F) \neq 1$ such that

$$
\|F\|_{\infty} \leq \sum_{\left(k_{1}, \cdots, k_{n}\right) \in S_{F}}\left\|\hat{F}\left(k_{1}, \cdots, k_{n}\right)\right\|_{M_{k}} \leq d(F)\|F\|_{\infty} .
$$

Proof. Let

$$
F\left(z_{1}, \cdots, z_{n}\right)=\sum_{\left(k_{1}, \cdots, k_{n}\right) \in S_{F}} \hat{F}\left(k_{1}, \cdots, k_{n}\right) z_{1}^{k_{1}} \cdots z_{n}^{k_{n}}
$$

be a $k \times k$-matrix of complex polynomials over $\mathbb{D}^{n}$. In general, we have

$$
\|F\|_{\infty} \leq \sum_{\left(k_{1}, \cdots, k_{n}\right) \in S_{F}}\left\|\hat{F}\left(k_{1}, \cdots, k_{n}\right)\right\|_{M_{k}}
$$

and

$$
\sum_{\left(k_{1}, \cdots, k_{n}\right) \in S_{F}}\left\|\hat{F}\left(k_{1}, \cdots, k_{n}\right)\right\|_{M_{k}} \in \mathbb{R}^{+},\|F\|_{\infty} \in \mathbb{R}^{+} .
$$

The Archimedean property of the total order on $\mathbb{R}$ allows us to claim that there exists a smallest positive integer $d(F) \neq 1$ such that

$$
\|F\|_{\infty} \leq \sum_{\left(k_{1}, \cdots, k_{n}\right) \in S_{F}}\left\|\hat{F}\left(k_{1}, \cdots, k_{n}\right)\right\|_{M_{k}} \leq d(F)\|F\|_{\infty} .
$$

Remark 2.3. Each element $F$ of the algebra $M_{k}\left(\mathcal{P}_{n}\right)$ has a finite number of Fourier coefficients and each Fourier coefficient of an element $F$ of $M_{k}\left(\mathcal{P}_{n}\right)$ is bounded by its supremum norm $\|F\|_{\infty}$ over the polydisc $\mathbb{D}^{n}$.

Let 


$$
F\left(z_{1}, \cdots, z_{n}\right)=\sum_{\left(k_{1}, \cdots, k_{n}\right) \in S_{F}} \hat{F}\left(k_{1}, \cdots, k_{n}\right) z_{1}^{k_{1}} \cdots z_{n}^{k_{n}}
$$

be an element of $M_{k}\left(\mathcal{P}_{n}\right)$ and let $\operatorname{Card}\left(S_{F}\right)$ be the number of the Fourier coefficients of $F$. Denote by

$$
\Omega_{k, r}\left(\mathcal{P}_{n}\right)=\left\{F \in M_{k}\left(\mathcal{P}_{n}\right): \operatorname{Card}\left(S_{F}\right)=r\right\} \subset M_{k}\left(\mathcal{P}_{n}\right) .
$$

Theorem 2.4. Let $\mathcal{P}_{n}$ be the set of complex polynomials over $\mathbb{D}^{n}$ and let

$$
\Omega_{k, r}\left(\mathcal{P}_{n}\right)=\left\{F \in M_{k}\left(\mathcal{P}_{n}\right): \operatorname{Card}\left(S_{F}\right)=r\right\}
$$

be a subspace of $M_{k}\left(\mathcal{P}_{n}\right)$. Then

$$
\sup \left\{\frac{\sum_{\left(k_{1}, \cdots, k_{n}\right) \in S_{F}}\left\|\hat{F}\left(k_{1}, \cdots, k_{n}\right)\right\|_{M_{k}}}{\|F\|_{\infty}}: F \in \Omega_{k, r}\left(\mathcal{P}_{n}\right), F \neq 0\right\} \leq r .
$$

Proof. Let

$$
F\left(z_{1}, \cdots, z_{n}\right)=\sum_{\left(k_{1}, \cdots, k_{n}\right) \in S_{F}} \hat{F}\left(k_{1}, \cdots, k_{n}\right) z_{1}^{k_{1}} \cdots z_{n}^{k_{n}}
$$

be an element of $\Omega_{k, r}\left(\mathcal{P}_{n}\right)$. Therefore, $\operatorname{Card}\left(S_{F}\right)=r$. Remark 2.3 allows us to claim that

$$
\left\|\hat{F}\left(k_{1}, \cdots, k_{n}\right)\right\|_{M_{k}} \leq\|F\|_{\infty}, \forall F \in M_{k}\left(\mathcal{P}_{n}\right),
$$

with

$$
F\left(z_{1}, \cdots, z_{n}\right)=\sum_{\left(k_{1}, \cdots, k_{n}\right) \in S_{F}} \hat{F}\left(k_{1}, \cdots, k_{n}\right) z_{1}^{k_{1}} \cdots z_{n}^{k_{n}} .
$$

It follows that

$$
\sum_{\left(k_{1}, \cdots, k_{n}\right) \in S_{F}}\left\|\hat{F}\left(k_{1}, \cdots, k_{n}\right)\right\|_{M_{k}} \leq \operatorname{Card}\left(S_{F}\right)\|F\|_{\infty}, \forall F \in M_{k}\left(\mathcal{P}_{n}\right) .
$$

For matrices of $\Omega_{k, r}\left(\mathcal{P}_{n}\right)$, we have the following:

$$
\sum_{\left(k_{1}, \cdots, k_{n}\right) \in S_{F}}\left\|\hat{F}\left(k_{1}, \cdots, k_{n}\right)\right\|_{M_{k}} \leq r\|F\|_{\infty}, \forall F \in \Omega_{k, r}\left(\mathcal{P}_{n}\right) .
$$

Therefore,

$$
\frac{\sum_{\left(k_{1}, \cdots, k_{n}\right) \in S_{F}}\left\|\hat{F}\left(k_{1}, \cdots, k_{n}\right)\right\|_{M_{k}}}{\|F\|_{\infty}} \leq r, \forall F \in \Omega_{k, r}\left(\mathcal{P}_{n}\right), F \neq 0 .
$$

Finally,

$$
\sup \left\{\frac{\sum_{\left(k_{1}, \cdots, k_{n}\right) \in S_{F}}\left\|\hat{F}\left(k_{1}, \cdots, k_{n}\right)\right\|_{M_{k}}}{\|F\|_{\infty}}, \forall F \in \Omega_{k, r}\left(\mathcal{P}_{n}\right), F \neq 0\right\} \leq r .
$$

Theorem 2.4 allows us to state that, for every $n$-tuple $\left\{T_{1}, \cdots, T_{n}\right\}$ of commuting contractions on a Hilbert space $\mathcal{H}$, one has

$$
\left\|F\left(T_{1}, \cdots, T_{n}\right)\right\|_{M_{k}} \leq r\|F\|_{\infty}, \forall F \in \Omega_{k, r}\left(\mathcal{P}_{n}\right)
$$


We can factorize matrices of complex polynomials. Assume that $n=3$ and let

$$
Q\left(z_{1}, z_{2}, z_{3}\right)=\sum_{\left(k_{1}, k_{2}, k_{3}\right) \in S_{F}} \hat{Q}\left(k_{1}, k_{2}, k_{3}\right) z_{1}^{k_{1}} z_{2}^{k_{2}} z_{3}^{k_{3}}
$$

be a matrix of complex polynomials of three variables of $M_{k}\left(\mathcal{P}_{3}\right)$. Then $\forall\left(k_{1}, k_{2}, k_{3}\right) \in S_{F}$ there exists a constant $\alpha\left(k_{1}, k_{2}, k_{3}\right) \in \mathbb{N}, \alpha\left(k_{1}, k_{2}, k_{3}\right) \neq 0$, such that

$$
\left\|2 \hat{Q}\left(k_{1}, k_{2}, k_{3}\right)\right\|_{M_{3}}<\left\|\left|2 \hat{Q}\left(k_{1}, k_{2}, k_{3}\right)+\alpha\left(k_{1}, k_{2}, k_{3}\right) I_{k}\right|^{2}\right\|_{M_{3}} .
$$

Let us notice that $\left|2 \hat{Q}\left(k_{1}, k_{2}, k_{3}\right)+\alpha\left(k_{1}, k_{2}, k_{3}\right) I_{k}\right|^{2}$ is a positive matrix of complex polynomials and $\alpha\left(k_{1}, k_{2}, k_{3}\right)$ is not unique. Denote by

$$
B_{1}\left(z_{1}, z_{2}, z_{3}\right)=\sum_{\left(k_{1}, k_{2}, k_{3}\right) \in S_{F}}\left|\hat{Q}\left(k_{1}, k_{2}, k_{3}\right)+\alpha\left(k_{1}, k_{2}, k_{3}\right) I_{k}\right|^{2}\left(z_{1}^{k_{1}} z_{2}^{k_{2}}+z_{1}^{k_{1}}\right)
$$

and

$$
B_{2}\left(z_{1}, z_{2}, z_{3}\right)=\sum_{\left(k_{1}, k_{2}, k_{3}\right) \in S_{F}}\left|\hat{Q}\left(k_{1}, k_{2}, k_{3}\right)+\alpha\left(k_{1}, k_{2}, k_{3}\right) I_{k}\right|^{2}\left(z_{2}^{k_{2}} z_{3}^{k_{3}}+z_{2}^{k_{2}}\right) .
$$

It is straightforward to see that

$$
\begin{aligned}
& \prod_{j=1}^{2} B_{j}\left(z_{1}, z_{2}, z_{3}\right) \\
& =\sum_{\left(k_{1}, k_{2}, k_{3}\right) \in S_{F}}\left|\hat{Q}\left(k_{1}, k_{2}, k_{3}\right)+\alpha\left(k_{1}, k_{2}, k_{3}\right) I_{k}\right|^{4} z_{1}^{k_{1}} z_{2}^{k_{2}} z_{3}^{k_{3}}+H\left(z_{1}, z_{2}, z_{3}\right) .
\end{aligned}
$$

This implies that there exists a matrix $P^{Q}\left(z_{1}, z_{2}, z_{3}\right) \in M_{k}\left(\mathcal{P}_{3}\right)$ of complex polynomials over $\mathbb{D}^{3}$ such that

$$
\prod_{j=1}^{2} B_{j}\left(Q\left(z_{1}, z_{2}, z_{3}\right)\right)=2 Q\left(z_{1}, z_{2}, z_{3}\right)+P^{Q}\left(z_{1}, z_{2}, z_{3}\right) .
$$

What we need to notice is that the sets

$$
\left\{\left\|F\left(T_{1}, \cdots, T_{n}\right)\right\|_{M_{k}}: F \in M_{k}\left(\mathcal{P}_{n}\right)\right\},\left\{\|F\|_{\infty}: F \in M_{k}\left(\mathcal{P}_{n}\right)\right\}
$$

are not bounded. However, the factorization of matrices of complex polynomials, in terms of the product of matrices of complex polynomials of two variables, allows us to claim that the set

$$
\left\{\frac{\left\|F\left(T_{1}, \cdots, T_{n}\right)\right\|_{M_{k}}}{\|F\|_{\infty}}: F \in M_{k}\left(\mathcal{P}_{n}\right), F \neq 0\right\}
$$

is bounded by $2^{n}$.

\section{Proof of Theorem 1.1}

Let

$$
F\left(z_{1}, \cdots, z_{n}\right)=\sum_{\left(k_{1}, \cdots, k_{n}\right) \in S_{F}} \hat{F}\left(k_{1}, \cdots, k_{n}\right) z_{1}^{k_{1}} \cdots z_{n}^{k_{n}} \in M_{k}\left(\mathcal{P}_{n}\right)
$$

be a matrix of complex polynomials over $\mathbb{D}^{n}$. Then $\forall\left(k_{1}, \cdots, k_{n}\right) \in S_{F}$ there exists a constant $\alpha_{2}\left(k_{1}, \cdots, k_{n}\right) \in \mathbb{N}, \alpha_{2}\left(k_{1}, \cdots, k_{n}\right) \neq 0$, such that 


$$
2\left\|\hat{F}\left(k_{1}, \cdots, k_{n}\right)\right\|_{M_{k}}<\left\|2 \hat{F}\left(k_{1}, \cdots, k_{n}\right)+\left.\alpha_{2}\left(k_{1}, \cdots, k_{n}\right) I_{k}\right|^{2}\right\|_{M_{k}} .
$$

Denote by

$$
\Delta^{(2)}\left(F\left(z_{1}, \cdots, z_{n}\right)\right)=\sum_{\left(k_{1}, \cdots, k_{n}\right) \in S_{F}}\left|2 \hat{F}\left(k_{1}, \cdots, k_{n}\right)+\alpha_{2}\left(k_{1}, \cdots, k_{n}\right) I_{k}\right|^{2} z_{1}^{k_{1}} \cdots z_{n}^{k_{n}}
$$

the matrix of complex polynomials with positive matrices as Fourier coefficients. The matrix of complex polynomials $\Delta^{(2)}\left(F\left(z_{1}, \cdots, z_{n}\right)\right)$ satisfies the von Neumann's inequality. Denote by

$$
\beta_{j, 2}\left(F\left(z_{1}, \cdots, z_{n}\right)\right)=\sum_{\left(k_{1}, \cdots, k_{n}\right) \in S_{F}}\left|2 \hat{F}\left(k_{1}, \cdots, k_{n}\right)+\alpha_{2}\left(k_{1}, \cdots, k_{n}\right) I_{k}\right|^{2} z_{j}^{k_{j}} z_{j+1}^{k_{j+1}}
$$

and

$$
\delta_{j, 2}\left(F\left(z_{1}, \cdots, z_{n}\right)\right)=\sum_{\left(k_{1}, \cdots, k_{n}\right) \in S_{F}}\left|2 \hat{F}\left(k_{1}, \cdots, k_{n}\right)+\alpha_{2}\left(k_{1}, \cdots, k_{n}\right) I_{k}\right|^{2} z_{j}^{k_{j}},
$$

$j=1, \cdots, n-1$. As we can see $\beta_{j, 2}\left(F\left(z_{1}, \cdots, z_{n}\right)\right), j=1, \cdots, n-1$, are matrices of complex polynomials of two variables with positive matrices as Fourier coefficients and $\delta_{j, 2}\left(F\left(z_{1}, \cdots, z_{n}\right)\right), j=1, \cdots, n-1$, are matrices of complex polynomials of one variable with positive matrices as Fourier coefficients. These polynomials have exactly the same number of Fourier coefficients than $F\left(z_{1}, \cdots, z_{n}\right)$ and they all satisfy the von Neumann inequality. It is straightforward to see that

$$
\begin{gathered}
\left\|\beta_{j, 2}(F)\right\|_{\infty}=\left\|\sum_{\left(k_{1}, \cdots, k_{n}\right) \in S_{F}}\left|2 \hat{F}\left(k_{1}, \cdots, k_{n}\right)+\alpha_{2}\left(k_{1}, \cdots, k_{n}\right) I_{k}\right|^{2}\right\|_{M_{k}}, \\
\left\|\delta_{j, 2}(F)\right\|_{\infty}=\left\|\beta_{j, 2}(F)\right\|_{\infty}
\end{gathered}
$$

and

$$
\left\|\beta_{j, 2}(F)+\delta_{j, 2}(F)\right\|_{\infty}=2\left\|\delta_{j, 2}(F)\right\|_{\infty}=2\left\|\beta_{j, 2}(F)\right\|_{\infty} .
$$

Denote by

$$
G_{j, 2}\left(F\left(z_{1}, \cdots, z_{n}\right)\right)=\beta_{j, 2}\left(F\left(z_{1}, \cdots, z_{n}\right)\right)+\delta_{j, 2}\left(F\left(z_{1}, \cdots, z_{n}\right)\right), j=1, \cdots, n-1 .
$$

That is,

$$
\begin{aligned}
& G_{j, 2}\left(F\left(z_{1}, \cdots, z_{n}\right)\right) \\
& =\sum_{\left(k_{1}, \cdots, k_{n}\right) \in S_{F}}\left|2 \hat{F}\left(k_{1}, \cdots, k_{n}\right)+\alpha_{2}\left(k_{1}, \cdots, k_{n}\right) I_{k}\right|^{2}\left(z_{j}^{k_{j}} z_{j+1}^{k_{j+1}}+z_{j}^{k_{j}}\right),
\end{aligned}
$$

$j=1, \cdots, n-1$. It is not difficult to see that there exists a matrix $P_{2}^{F}\left(z_{1}, \cdots, z_{n}\right) \in M_{k}\left(\mathcal{P}_{n}\right)$ of complex polynomials over $\mathbb{D}^{n}$ such that

$$
\prod_{j=1}^{n-1} G_{j, 2}\left(F\left(z_{1}, \cdots, z_{n}\right)\right)=2 F\left(z_{1}, \cdots, z_{n}\right)+P_{2}^{F}\left(z_{1}, \cdots, z_{n}\right) .
$$

In other words,

$$
2 F\left(z_{1}, \cdots, z_{n}\right)+P_{2}^{F}\left(z_{1}, \cdots, z_{n}\right)=\prod_{j=1}^{n-1}\left[\beta_{j, 2}\left(F\left(z_{1}, \cdots, z_{n}\right)\right)+\delta_{j, 2}\left(F\left(z_{1}, \cdots, z_{n}\right)\right)\right] .
$$


It is straightforward to see that

$$
\left\|\prod_{j=1}^{n-1} G_{2, j}(F)\right\|_{\infty}=\left\|2 F+P_{2}^{F}\right\|_{\infty}=\prod_{j=1}^{n-1}\left\|\beta_{j, 2}(F)+\delta_{j, 2}(F)\right\|_{\infty} .
$$

In general, the matrix $P_{2}^{F}\left(z_{1}, \cdots, z_{n}\right)$ is not unique. The structure of the matrix $P_{2}^{F}\left(z_{1}, \cdots, z_{n}\right)$ depends on the structure of $\Delta^{(2)}\left(F\left(z_{1}, \cdots, z_{n}\right)\right)$. Let $\left\{T_{1}, T_{2}, \cdots, T_{n}\right\}$ be an $n$-tuple of commuting contractions on a Hilbert space $\mathcal{H}$. The von Neumann inequality for matrices of complex polynomials of two (or one) variable(s) allows us to say that

$$
\frac{\left\|\beta_{j, 2}\left(F\left(T_{1}, \cdots, T_{n}\right)\right)\right\|}{\left\|\beta_{j, 2}(F)\right\|_{\infty}} \leq 1, \frac{\left\|\delta_{j, 2}\left(F\left(T_{1}, \cdots, T_{n}\right)\right)\right\|}{\left\|\delta_{j, 2}(F)\right\|_{\infty}} \leq 1 .
$$

This implies that

$$
\frac{\left\|\beta_{j, 2}\left(F\left(T_{1}, \cdots, T_{n}\right)\right)\right\|}{\left\|\beta_{j, 2}(F)\right\|_{\infty}}+\frac{\left\|\delta_{j, 2}\left(F\left(T_{1}, \cdots, T_{n}\right)\right)\right\|}{\left\|\delta_{j, 2}(F)\right\|_{\infty}} \leq 2 .
$$

Suppose that

$$
G_{2}^{F}\left(z_{1}, \cdots, z_{n}\right)=\prod_{j=1}^{n-1} G_{j}\left(F\left(z_{1}, \cdots, z_{n}\right)\right)=2 F\left(z_{1}, \cdots, z_{n}\right)+P_{2}^{F}\left(z_{1}, \cdots, z_{n}\right) .
$$

It follows that

$$
G_{2}^{F}\left(T_{1}, \cdots, T_{n}\right)=\prod_{j=1}^{n-1}\left[\beta_{j, 2}\left(F\left(T_{1}, \cdots, T_{n}\right)\right)+\delta_{j, 2}\left(F\left(T_{1}, \cdots, T_{n}\right)\right)\right] .
$$

This implies that,

$$
\left\|G_{2}^{F}\left(T_{1}, \cdots, T_{n}\right)\right\|=\left\|\prod_{j=1}^{n-1}\left[\beta_{j, 2}\left(F\left(T_{1}, \cdots, T_{n}\right)\right)+\delta_{j, 2}\left(F\left(T_{1}, \cdots, T_{n}\right)\right)\right]\right\| .
$$

Thus,

$$
\left\|G_{2}^{F}\left(T_{1}, \cdots, T_{n}\right)\right\| \leq \prod_{j=1}^{n-1}\left\|\left[\beta_{j, 2}\left(F\left(T_{1}, \cdots, T_{n}\right)\right)+\delta_{j, 2}\left(F\left(T_{1}, \cdots, T_{n}\right)\right)\right]\right\| .
$$

Also, we have

$$
\left\|G_{2}^{F}\left(T_{1}, \cdots, T_{n}\right)\right\| \leq \prod_{j=1}^{n-1}\left[\left\|\beta_{j, 2}\left(F\left(T_{1}, \cdots, T_{n}\right)\right)\right\|+\left\|\delta_{j, 2}\left(F\left(T_{1}, \cdots, T_{n}\right)\right)\right\|\right] .
$$

It is easy to say that

$$
\frac{\left\|G_{2}^{F}\left(T_{1}, \cdots, T_{n}\right)\right\|}{\left\|G_{2}^{F}\right\|_{\infty}} \leq \prod_{j=1}^{n-1}\left[\frac{\left\|\beta_{j, 2}\left(F\left(T_{1}, \cdots, T_{n}\right)\right)\right\|+\left\|\delta_{j, 2}\left(F\left(T_{1}, \cdots, T_{n}\right)\right)\right\|}{\left\|\beta_{j, 2}(F)+\delta_{j, 2}(F)\right\|_{\infty}}\right] .
$$

Therefore,

$$
\frac{\left\|G_{2}^{F}\left(T_{1}, \cdots, T_{n}\right)\right\|}{\left\|G_{2}^{F}\right\|_{\infty}} \leq \prod_{j=1}^{n-1}\left[\frac{\left\|\beta_{j, 2}\left(F\left(T_{1}, \cdots, T_{n}\right)\right)\right\|}{\left\|\beta_{j, 2}(F)+\delta_{j, 2}(F)\right\|_{\infty}}+\frac{\left\|\delta_{j, 2}\left(F\left(T_{1}, \cdots, T_{n}\right)\right)\right\|}{\left\|\beta_{j, 2}(F)+\delta_{j, 2}(F)\right\|_{\infty}}\right] .
$$

We can claim that 


$$
\frac{\left\|G_{2}^{F}\left(T_{1}, \cdots, T_{n}\right)\right\|}{\left\|G_{2}^{F}\right\|_{\infty}} \leq \prod_{j=1}^{n-1}\left[\frac{\left\|\beta_{j, 2}\left(F\left(T_{1}, \cdots, T_{n}\right)\right)\right\|}{\left\|\beta_{j, 2}(F)\right\|_{\infty}}+\frac{\left\|\delta_{j, 2}\left(F\left(T_{1}, \cdots, T_{n}\right)\right)\right\|}{\left\|\delta_{j, 2}(F)\right\|_{\infty}}\right],
$$

since, in general,

$$
\left\|\delta_{j, 2}(F)\right\|_{\infty} \leq\left\|\beta_{j, 2}(F)+\delta_{j, 2}(F)\right\|_{\infty},\left\|\beta_{j, 2}(F)\right\|_{\infty} \leq\left\|\beta_{j, 2}(F)+\delta_{j, 2}(F)\right\|_{\infty} .
$$

It is clear that

$$
\frac{\left\|G_{2}^{F}\left(T_{1}, \cdots, T_{n}\right)\right\|}{\left\|G_{2}^{F}\right\|_{\infty}} \leq \prod_{j=1}^{n-1}[1+1]=2^{n-1} .
$$

This implies that

$$
\sup \left\{\frac{\left\|2 F\left(T_{1}, \cdots, T_{n}\right)+P_{2}^{F}\left(T_{1}, \cdots, T_{n}\right)\right\|}{\left\|2 F+P_{2}^{F}\right\|_{\infty}}: F \in M_{k}\left(\mathcal{P}_{n}\right), F \neq 0\right\} \leq 2^{n-1} .
$$

Due to the fact that

$$
\left\|2 F\left(T_{1}, \cdots, T_{n}\right)+P_{2}^{F}\left(T_{1}, \cdots, T_{n}\right)\right\| \leq\left\|2 F\left(T_{1}, \cdots, T_{n}\right)\right\|+\left\|P_{2}^{F}\left(T_{1}, \cdots, T_{n}\right)\right\|
$$

and

$$
\left\|2 F+P_{2}^{F}\right\|_{\infty} \leq\|2 F\|_{\infty}+\left\|P_{2}^{F}\right\|_{\infty},
$$

we can say that for every $F \in M_{k}\left(\mathcal{P}_{n}\right), F \neq 0$, there exist two positive real numbers $\alpha_{F}^{V}$ and $\lambda_{F}$ such that

$$
\left\|2 F\left(T_{1}, \cdots, T_{n}\right)+P_{2}^{F}\left(T_{1}, \cdots, T_{n}\right)\right\|+\alpha_{F, 2}^{V}=\left\|2 F\left(T_{1}, \cdots, T_{n}\right)\right\|+\left\|P_{2}^{F}\left(T_{1}, \cdots, T_{n}\right)\right\|
$$

and

$$
\left\|2 F+P_{2}^{F}\right\|_{\infty}+\lambda_{F, 2}=\|2 F\|_{\infty}+\left\|P_{2}^{F}\right\|_{\infty} .
$$

Thus,

$$
\left\|2 F\left(T_{1}, \cdots, T_{n}\right)+P_{2}^{F}\left(T_{1}, \cdots, T_{n}\right)\right\|=\left\|2 F\left(T_{1}, \cdots, T_{n}\right)\right\|+\left\|P_{2}^{F}\left(T_{1}, \cdots, T_{n}\right)\right\|-\alpha_{F, 2}^{V}
$$

and

$$
\left\|2 F+P_{2}^{F}\right\|_{\infty}=\|2 F\|_{\infty}+\left\|P_{2}^{F}\right\|_{\infty}-\lambda_{F, 2} .
$$

Therefore,

$$
\frac{\left\|2 F\left(T_{1}, \cdots, T_{n}\right)+P_{2}^{F}\left(T_{1}, \cdots, T_{n}\right)\right\|}{\left\|2 F+P_{2}^{F}\right\|_{\infty}}=\frac{\left\|2 F\left(T_{1}, \cdots, T_{n}\right)\right\|+\left\|P_{2}^{F}\left(T_{1}, \cdots, T_{n}\right)\right\|-\alpha_{F, 2}^{V}}{\|2 F\|_{\infty}+\left\|P_{2}^{F}\right\|_{\infty}-\lambda_{F, 2}} .
$$

It is not difficult to see that

$$
\frac{\left\|2 F\left(T_{1}, \cdots, T_{n}\right)\right\|+\left\|P_{2}^{F}\left(T_{1}, \cdots, T_{n}\right)\right\|-\alpha_{F, 2}^{V}}{\|2 F\|_{\infty}+\left\|P_{2}^{F}\right\|_{\infty}} \leq \frac{\left\|2 F\left(T_{1}, \cdots, T_{n}\right)+P_{2}^{F}\left(T_{1}, \cdots, T_{n}\right)\right\|}{\left\|2 F+P_{2}^{F}\right\|_{\infty}}
$$

Now, we can claim that

$$
\frac{\left\|2 F\left(T_{1}, \cdots, T_{n}\right)\right\|+\left\|P_{2}^{F}\left(T_{1}, \cdots, T_{n}\right)\right\|-\alpha_{F, 2}^{V}}{\|2 F\|_{\infty}+\left\|P_{2}^{F}\right\|_{\infty}} \leq 2^{n-1}, F \in M_{k}\left(\mathcal{P}_{n}\right), F \neq 0 .
$$


There exists a positive real number $\theta_{P_{2}^{F}}^{V}$ such that

$$
\left\|P_{2}^{F}\left(T_{1}, \cdots, T_{n}\right)\right\|+\theta_{P_{2}^{F}}^{V}=\sum_{\left(k_{1}, \cdots, k_{n}\right) \in S_{P_{2}^{F}}^{F}}\left\|\hat{P}_{2}^{F}\left(k_{1}, \cdots, k_{n}\right)\right\|_{M_{k}} .
$$

This allows us to say that

$$
\frac{\left\|2 F\left(T_{1}, \cdots, T_{n}\right)\right\|+\sum_{\left(k_{1}, \cdots, k_{n}\right) \in S_{P_{2}^{F}}}\left\|\hat{P}_{2}^{F}\left(k_{1}, \cdots, k_{n}\right)\right\|_{M_{k}}-\theta_{P_{2}^{F}}^{V}-\alpha_{F, 2}^{V}}{\|2 F\|_{\infty}+\left\|P_{2}^{F}\right\|_{\infty}} \leq 2^{n-1} .
$$

In other words,

$$
\frac{\left\|2 F\left(T_{1}, \cdots, T_{n}\right)\right\|+\sum_{\left(k_{1}, \cdots, k_{n}\right) \in S_{P_{2}^{F}}}\left\|\hat{P}_{2}^{F}\left(k_{1}, \cdots, k_{n}\right)\right\|_{M_{k}}-\omega_{F, 2}^{V}}{\|2 F\|_{\infty}+\left\|P_{2}^{F}\right\|_{\infty}} \leq 2^{n-1} .
$$

with $\omega_{F, 2}^{V}=\theta_{P^{F}}^{V}+\alpha_{F, 2}^{V} \geq 0$. Let us notice that

$$
0 \leq \omega_{F, 2}^{V} \leq 2\left\|F\left(T_{1}, \cdots, T_{n}\right)\right\|+\sum_{\left(k_{1}, \cdots, k_{n}\right) \in S_{P_{2}^{F}}}\left\|\hat{P}_{2}^{F}\left(k_{1}, \cdots, k_{n}\right)\right\|_{M_{k}}, F \in M_{k}\left(\mathcal{P}_{n}\right), F \neq 0,
$$

and

$$
0 \leq \frac{\omega_{F, 2}^{V}}{2\|F\|_{\infty}+\left\|P_{2}^{F}\right\|_{\infty}} \leq 2^{n-1}, F \in M_{k}\left(\mathcal{P}_{n}\right), F \neq 0 .
$$

It is straightforward to observe that if we add (2.1) and (2.2), one has

$$
\leq \frac{\left\|2 F\left(T_{1}, \cdots, T_{n}\right)\right\|+\sum_{\left(k_{1}, \cdots, k_{n}\right) \in S_{P_{2}^{F}}}\left\|\hat{P}_{2}^{F}\left(k_{1}, \cdots, k_{n}\right)\right\|_{M_{k}}}{\|2 F\|_{\infty}+\left\|P_{2}^{F}\right\|_{\infty}} \leq 2^{n-1}+2^{n-1},
$$

$F \in M_{k}\left(\mathcal{P}_{n}\right), F \neq 0$. It follows that

$$
0 \leq \frac{\left\|2 F\left(T_{1}, \cdots, T_{n}\right)\right\|+\left\|P_{2}^{F}\right\|_{\infty}}{\|2 F\|_{\infty}+\left\|P_{2}^{F}\right\|_{\infty}} \leq 2^{n}, \forall F \in M_{k}\left(\mathcal{P}_{n}\right), F \neq 0 .
$$

Therefore, there exists a positive constant $K_{n}$ such that

$$
0 \leq \frac{\left\|F\left(T_{1}, \cdots, T_{n}\right)\right\|}{\|F\|_{\infty}} \leq K_{n}, \forall F \in M_{k}\left(\mathcal{P}_{n}\right), F \neq 0 .
$$

Define the sequence $\left(\Delta^{(m)}\left(F\left(z_{1}, \cdots, z_{n}\right)\right)\right)_{m \in \mathbb{N}, m \neq 0}$ of matrices of complex polynomials over $\mathbb{D}^{n}$ by setting

$$
\Delta^{(m)}\left(F\left(z_{1}, \cdots, z_{n}\right)\right)=\sum_{\left(k_{1}, \cdots, k_{n}\right) \in S_{F}}\left|m \hat{F}\left(k_{1}, \cdots, k_{n}\right)+\alpha_{m}\left(k_{1}, \cdots, k_{n}\right) I_{k}\right|^{2} z_{1}^{k_{1}} \cdots z_{n}^{k_{n}},
$$

with $\alpha_{m}\left(k_{1}, \cdots, k_{n}\right) \in \mathbb{N}, \alpha_{m}\left(k_{1}, \cdots, k_{n}\right) \neq 0$, such that

$$
m\left\|\hat{F}\left(k_{1}, \cdots, k_{n}\right)\right\|_{M_{k}}<\left\|m \hat{F}\left(k_{1}, \cdots, k_{n}\right)+\left.\alpha_{2}\left(k_{1}, \cdots, k_{n}\right) I_{k}\right|^{2}\right\|_{M_{k}} .
$$

The elements of the sequence $\left(\Delta^{(m)}\left(F\left(z_{1}, \cdots, z_{n}\right)\right)\right)_{m \in \mathbb{N}, m \neq 0}$ satisfy the von 
Neumann inequality. Let

$$
\left(\beta_{j, m}\left(F\left(z_{1}, \cdots, z_{n}\right)\right)\right)_{m \in \mathbb{N}, m \neq 0},\left(\delta_{j, m}\left(F\left(z_{1}, \cdots, z_{n}\right)\right)\right)_{m \in \mathbb{N}, m \neq 0}
$$

be two new sequences of matrices of complex polynomials over $\mathbb{D}^{n}$ defined by

$$
\beta_{j, m}\left(F\left(z_{1}, \cdots, z_{n}\right)\right)=\sum_{\left(k_{1}, \cdots, k_{n}\right) \in S_{F}}\left|m \hat{F}\left(k_{1}, \cdots, k_{n}\right)+\alpha_{m}\left(k_{1}, \cdots, k_{n}\right) I_{k}\right|^{2} z_{j}^{k_{j}} z_{j+1}^{k_{j+1}}
$$

and

$$
\begin{aligned}
& \quad \delta_{j, m}\left(F\left(z_{1}, \cdots, z_{n}\right)\right)=\sum_{\left(k_{1}, \cdots, k_{n}\right) \in S_{F}}\left|m \hat{F}\left(k_{1}, \cdots, k_{n}\right)+\alpha_{m}\left(k_{1}, \cdots, k_{n}\right) I_{k}\right|^{2} z_{j}^{k_{j}}, \\
& j=1, \cdots, n-1 . \text { For every } m \in \mathbb{N}, m \neq 0, \text { one has }
\end{aligned}
$$

$$
\frac{\left\|\beta_{j, m}\left(F\left(T_{1}, \cdots, T_{n}\right)\right)\right\|}{\left\|\beta_{j, m}(F)\right\|_{\infty}} \leq 1, \frac{\left\|\delta_{j, m}\left(F\left(T_{1}, \cdots, T_{n}\right)\right)\right\|}{\left\|\delta_{j, m}(F)\right\|_{\infty}} \leq 1 .
$$

A straightforward calculation shows that there exists a new sequence $\left(P_{m}^{F}\left(z_{1}, \cdots, z_{n}\right)\right)_{m \in \mathbb{N}, m \neq 0}$ of matrices of complex polynomials over $\mathbb{D}^{n}$ such that $m F\left(z_{1}, \cdots, z_{n}\right)+P_{m}^{F}\left(z_{1}, \cdots, z_{n}\right)=\prod_{j=1}^{n-1}\left[\beta_{j, m}\left(F\left(z_{1}, \cdots, z_{n}\right)\right)+\delta_{j, m}\left(F\left(z_{1}, \cdots, z_{n}\right)\right)\right]$.

We have exactly the same result as in (2.4). In other words,

$$
0 \leq \frac{m\left\|F\left(T_{1}, \cdots, T_{n}\right)\right\|+\left\|P_{m}^{F}\right\|_{\infty}}{m\|F\|_{\infty}+\left\|P_{m}^{F}\right\|_{\infty}} \leq 2^{n}, \forall F \in M_{k}\left(\mathcal{P}_{n}\right), F \neq 0 .
$$

Denote by $\alpha_{m}(F)=\left\|P_{m}^{F}\right\|_{\infty}$. Finally, for every $F \in M_{k}\left(\mathcal{P}_{n}\right)$, there exists a sequence $\left(\alpha_{m}(F)\right)_{m \in \mathbb{N}, m \neq 0}$ of positive numbers such that

$$
0 \leq \frac{m\left\|F\left(T_{1}, \cdots, T_{n}\right)\right\|+\alpha_{m}(F)}{m\|F\|_{\infty}+\alpha_{m}(F)} \leq 2^{n}, \forall F \in M_{k}\left(\mathcal{P}_{n}\right), F \neq 0 .
$$

Let us notice that the constant $\alpha_{m}(F)$ depends only on the Fourier coefficients of the matrix of complex polynomials $F\left(z_{1}, \cdots, z_{n}\right)$. Let us estimate the growth of $\alpha_{m}(F)$ depending on the values of $m$. Let us examine the number of terms of the product

$$
\prod_{j=1}^{n-1}\left[\beta_{j, m}\left(F\left(z_{1}, \cdots, z_{n}\right)\right)+\delta_{j, m}\left(F\left(z_{1}, \cdots, z_{n}\right)\right)\right] .
$$

This matrix of complex polynomials has exactly $2^{n-1}$ terms and there exists a matrix of complex polynomials $Q_{m}^{F}$

$$
m F\left(z_{1}, \cdots, z_{n}\right)+Q_{m}^{F}\left(z_{1}, \cdots, z_{n}\right)=\prod_{j=1}^{n-2} \delta_{j, m}\left(F\left(z_{1}, \cdots, z_{n}\right)\right) \beta_{n-1, m}\left(F\left(z_{1}, \cdots, z_{n}\right)\right) .
$$

A simple calculation shows that

$$
\left\|\prod_{j=1}^{n-1}\left[\beta_{j, m}(F)+\delta_{j, m}(F)\right]\right\|_{\infty}=2^{n-1}\left\|\delta_{1, m}(F)\right\|_{\infty}^{n-1} .
$$

In other words, 


$$
\begin{aligned}
& \left\|\prod_{j=1}^{n-1}\left[\beta_{j, m}(F)+\delta_{j, m}(F)\right]\right\|_{\infty} \\
& =2^{n-1}\left\|\sum_{\left(k_{1}, \cdots, k_{n}\right) \in S_{F}}\left|m \hat{F}\left(k_{1}, \cdots, k_{n}\right)+\alpha_{m}\left(k_{1}, \cdots, k_{n}\right)\right|^{2}\right\|_{M_{k}}^{n-1} .
\end{aligned}
$$

The expression of $m F\left(z_{1}, \cdots, z_{n}\right)$ can be deduced from the matrix of complex polynomials

$$
\prod_{j=1}^{n-2} \delta_{j, m}\left(F\left(z_{1}, \cdots, z_{n}\right)\right) \beta_{n-1, m}\left(F\left(z_{1}, \cdots, z_{n}\right)\right) .
$$

This means that the expression of the matrix $P_{m}^{F}\left(z_{1}, \cdots, z_{n}\right)$ can be computed from the $2^{n-1}-1$ remaining terms of the matrix of complex polynomials

$$
\prod_{j=1}^{n-1}\left[\beta_{j, m}\left(F\left(z_{1}, \cdots, z_{n}\right)\right)+\delta_{j, m}\left(F\left(z_{1}, \cdots, z_{n}\right)\right)\right] .
$$

It follows that

$$
\left\|P_{m}^{F}\right\|_{\infty}=\left(2^{n-1}-1\right)\left\|\sum_{\left(k_{1}, \cdots, k_{n}\right) \in S_{F}}\left|m \hat{F}\left(k_{1}, \cdots, k_{n}\right)+\alpha_{m}\left(k_{1}, \cdots, k_{n}\right)\right|^{2}\right\|_{M_{k}}^{n-1}+\varphi_{m}(F),
$$

with

$$
\sum_{\left(k_{1}, \cdots, k_{n}\right) \in S_{F}} m\left\|\hat{F}\left(k_{1}, \cdots, k_{n}\right)\right\|_{M_{k}}<\varphi_{m}(F)<\left\|\prod_{j=1}^{n-2} \delta_{j, m}(F) \beta_{n-1, m}(F)\right\|_{\infty} .
$$

Recall that

$$
\left\|\prod_{j=1}^{n-2} \delta_{j, m}(F) \beta_{n-1, m}(F)\right\|_{\infty}=\left\|\sum_{\left(k_{1}, \cdots, k_{n}\right) \in S_{F}}\left|m \hat{F}\left(k_{1}, \cdots, k_{n}\right)+\alpha_{m}\left(k_{1}, \cdots, k_{n}\right)\right|^{2}\right\|_{M_{k}}^{n-1} .
$$

Therefore, we can claim that

$$
\varphi_{m}(F)<\left\|\sum_{\left(k_{1}, \cdots, k_{n}\right) \in S_{F}}\left|m \hat{F}\left(k_{1}, \cdots, k_{n}\right)+\alpha_{m}\left(k_{1}, \cdots, k_{n}\right)\right|^{2}\right\|_{M_{k}}^{n-1} .
$$

\section{Application}

In this section, we show that von Neumann's inequality holds up to the constant $2^{n}$.

Remark 3.1. Let

$$
F\left(z_{1}, \cdots, z_{n}\right)=\sum_{\left(k_{1}, \cdots, k_{n}\right) \in S_{F}} \hat{F}\left(k_{1}, \cdots, k_{n}\right) z_{1}^{k_{1}} \cdots z_{n}^{k_{n}} \in M_{k}\left(\mathcal{P}_{n}\right)
$$

be a matrix of complex polynomials over $\mathbb{D}^{n}$. There exists a positive constant $\lambda_{m}(F)$ such that

$$
\left\|\sum_{\left(k_{1}, \cdots, k_{n}\right) \in S_{F}}\left|m \hat{F}\left(k_{1}, \cdots, k_{n}\right)+\alpha_{m}\left(k_{1}, \cdots, k_{n}\right)\right|^{2}\right\|_{M_{k}}^{n-1}=m\|F\|_{\infty}+\lambda_{m}(F)
$$

and 


$$
m\|F\|_{\infty}<\frac{\lambda_{m}(F)}{2},
$$

where $\alpha_{m}\left(k_{1}, \cdots, k_{n}\right) \neq 0$ is a positive integer such that

$$
\left\|m \hat{F}\left(k_{1}, \cdots, k_{n}\right)\right\|_{M_{k}}<\left\|m \hat{F}\left(k_{1}, \cdots, k_{n}\right)+\left.\alpha_{m}\left(k_{1}, \cdots, k_{n}\right)\right|^{2}\right\|_{M_{k}} .
$$

We can now show that von Neumann's inequality hold up to the constant $2^{n}$.

Theorem 3.2. Let $\mathcal{P}_{n}$ be the algebra of complex polynomials over $\mathbb{D}^{n}$, let

$$
F\left(z_{1}, \cdots, z_{n}\right)=\sum_{\left(k_{1}, \cdots, k_{n}\right) \in S_{F}} \hat{F}\left(k_{1}, \cdots, k_{n}\right) z_{1}^{k_{1}} \cdots z_{n}^{k_{n}} \in M_{k}\left(\mathcal{P}_{n}\right)
$$

be a matrix of complex polynomials over $\mathbb{D}^{n}$ and let $\left\{T_{1}, \cdots, T_{n}\right\}$ be an n-tuple of commuting contractions on a Hilbert space $\mathcal{H}$. Then

$$
\left\|F\left(T_{1}, \cdots, T_{n}\right)\right\|<\sum_{\left(k_{1}, \cdots, k_{n}\right) \in S_{F}}\left\|\hat{F}\left(k_{1}, \cdots, k_{n}\right)\right\|_{M_{k}} \leq 2^{n}\|F\|_{\infty}, \forall F \in M_{k}\left(\mathcal{P}_{n}\right) .
$$

Proof. Let

$$
F\left(z_{1}, \cdots, z_{n}\right)=\sum_{\left(k_{1}, \cdots, k_{n}\right) \in S_{F}} \hat{F}\left(k_{1}, \cdots, k_{n}\right) z_{1}^{k_{1}} \cdots z_{n}^{k_{n}} \in M_{k}\left(\mathcal{P}_{n}\right)
$$

be a matrix of complex polynomials over $\mathbb{D}^{n}$. Theorem 1.1 allows us to claim that there exists a sequence $\left(P_{m}^{F}\left(z_{1}, \cdots, z_{n}\right)\right)_{m \in \mathbb{N}, m \neq 0}$ of matrices of complex polynomials over $\mathbb{D}^{n}$ such that

$$
1<\left\|P_{m}^{F}\right\|_{\infty}<2^{n-1}\left\|\sum_{\left(k_{1}, \cdots, k_{n}\right) \in S_{F}}\left|m \hat{F}\left(k_{1}, \cdots, k_{n}\right)+\alpha_{m}\left(k_{1}, \cdots, k_{n}\right)\right|^{2}\right\|_{M_{k}}^{n-1},
$$

where $\alpha_{m}\left(k_{1}, \cdots, k_{n}\right) \neq 0$ is a positive integer such that

$$
\left\|m \hat{F}\left(k_{1}, \cdots, k_{n}\right)\right\|_{M_{k}}<\left\|m \hat{F}\left(k_{1}, \cdots, k_{n}\right)+\left.\alpha_{m}\left(k_{1}, \cdots, k_{n}\right)\right|^{2}\right\|_{M_{k}} .
$$

Recall that

$$
(a+b)^{n-1}=\sum_{k=0}^{n-1} \frac{(n-1) !}{(n-1-k) ! k !} a^{k} b^{n-1-k} .
$$

Remark 3.1 allows us to claim that there exists a positive constant $\lambda_{m}(F)$ such that

$$
\left\|\sum_{\left(k_{1}, \cdots, k_{n}\right) \in S_{F}}\left|m \hat{F}\left(k_{1}, \cdots, k_{n}\right)+\alpha_{m}\left(k_{1}, \cdots, k_{n}\right)\right|^{2}\right\|_{M_{k}}^{n-1}=m\|F\|_{\infty}+\lambda_{m}(F)
$$

and

$$
m\|F\|_{\infty}<\frac{\lambda_{m}(F)}{2}
$$

It follows that

$$
\begin{aligned}
& \left(2^{n-1}-1\right)\left(m\|F\|_{\infty}+\lambda_{m}(F)\right)+\sum_{\left(k_{1}, \cdots, k_{n}\right) \in S_{F}} m\left\|\hat{F}\left(k_{1}, \cdots, k_{n}\right)\right\|_{M_{k}} \\
& <2^{n-1}\left(m\|F\|_{\infty}+\lambda_{m}(F)\right) .
\end{aligned}
$$


This implies that

$$
2^{n-1}\left(m\|F\|_{\infty}+\lambda_{m}(F)\right)+\sum_{\left(k_{1}, \cdots, k_{n}\right) \in S_{F}} m\left\|\hat{F}\left(k_{1}, \cdots, k_{n}\right)\right\|_{M_{k}}<2^{n}\left(m\|F\|_{\infty}+\lambda_{m}(F)\right) .
$$

Therefore,

$$
\frac{2^{n}\left(m\|F\|_{\infty}+\lambda_{m}(F)\right)}{2}+\sum_{\left(k_{1}, \cdots, k_{n}\right) \in S_{F}} m\left\|\hat{F}\left(k_{1}, \cdots, k_{n}\right)\right\|_{M_{k}}<2^{n}\left(m\|F\|_{\infty}+\lambda_{m}(F)\right) .
$$

Due to the fact that $m\|F\|_{\infty}<\frac{\lambda_{m}(F)}{2}$, implies that

$$
\frac{2^{n}\|m F\|_{\infty}}{2}+\sum_{\left(k_{1}, \cdots, k_{n}\right) \in S_{F}} m\left\|\hat{F}\left(k_{1}, \cdots, k_{n}\right)\right\|_{M_{k}}<2^{n}\|m F\|_{\infty}, \forall F \in M_{k}\left(\mathcal{P}_{n}\right) \text {. }
$$

Therefore,

$$
\sum_{\left(k_{1}, \cdots, k_{n}\right) \in S_{F}}\left\|\hat{F}\left(k_{1}, \cdots, k_{n}\right)\right\|_{M_{k}}<2^{n}\|F\|_{\infty}, \forall F \in M_{k}\left(\mathcal{P}_{n}\right) .
$$

Finally, if $\left\{T_{1}, \cdots, T_{n}\right\}$ is an $n$-tuple of commuting contractions on a Hilbert space $\mathcal{P}$, we have

$$
\left\|f\left(T_{1}, \cdots, T_{n}\right)\right\|<\sum_{\left(k_{1}, \cdots, k_{n}\right) \in S_{F}}\left\|\hat{F}\left(k_{1}, \cdots, k_{n}\right)\right\|_{M_{k}}<2^{n}\|F\|_{\infty}, \forall F \in M_{k}\left(\mathcal{P}_{n}\right) .
$$

We also notice that the fraction $\frac{\left\|F\left(T_{1}, \cdots, T_{n}\right)\right\|}{\|F\|_{\infty}}, \forall F \in M_{k}\left(\mathcal{P}_{n}\right), \quad F \neq 0$, depends on the expression of $\mathrm{F}$ not on the number of variables of $F$. However, the constant $2^{n}$ depends on the number of variables of $F$. This means that for the sufficiently large number of variables, the inequality (2.6) is trivial.

\section{Conflicts of Interest}

The authors declare no conflicts of interest regarding the publication of this paper.

\section{References}

[1] von Neumann, J. (1951) Eine Spektraltheorie für allgemeine Operatoren eines unitären Raumes. Mathematische Nachrichten, 4, 258-281.

https://doi.org/10.1002/mana.3210040124

[2] Brehmer, S. (1961) Uber vertauschbare Kontractionen des Hilbertschen Raumes. Acta Scientiarum Mathematicarum (Szeged), 22, 106-111.

[3] Ando, T. (1963) On a Pair of Commutative Contractions. Acta Scientiarum Mathematicarum (Szeged), 24, 88-90.

[4] Varopoulos, N.T. (1974) On an Inequality of Von Neumann and an Application of the Metric Theory of Tensor Products to Operators Theory. Journal of Functional Analysis, 16, 83-100. https://doi.org/10.1016/0022-1236(74)90071-8

[5] Paulsen, V.I. (1986) Completely Bounded Maps and Dilations. London Mathematical Society, Harlow, Essex, England.

[6] Lubin, A. (1978) Research Notes on Von Neumann's Inequality. International Journal 
of Mathematics and Mathematical Sciences, 1, 133-136. https://doi.org/10.1155/S0161171278000162

[7] Nikolski, N.K. (2002) Operators, Functions, and Systems: An Easy Reading, Vol. 2: Model Operators and Systems. American Mathematical Society, USA.

[8] Kosiński, L. (2015) Three-Point Nevanlinna-Pick Problem in the Polydisc. Proceedings of the London Mathematical Society, 111, 887-910. https://doi.org/10.1112/plms/pdv045

[9] Moussounda Mouanda, J. (2020) On Von Neumann's Inequality for Tuples of Complex Triangular Toeplitz Contractions. Rocky Mountain Journal of Mathematics, 50, 213-224. https://doi.org/10.1216/rmj.2020.50.213

[10] Moussounda Mouanda, J. (2021) On Kadison's Similary Problem for Homomorphism of the Algebra of Complex Polynomials. Advances in Pure Mathematics, 11, 755-770. https://doi.org/10.4236/apm.2021.119050

[11] Lotto, B.A. (1994) Von Neumann's Inequality for Commuting, Diagonalizable Contractions, I. Proceedings of the American Mathematical Society, 120, 889-895.

https://doi.org/10.1090/S0002-9939-1994-1169881-8 